\section{The Correlation of Sorted Scores Rule}

\author{
Kimmo Sorjonen ${ }^{1}$, Fredrik Ullén ${ }^{2}, \&$ Bo Melin ${ }^{1}$
}

Karolinska Intitutet, Solna, Sweden

${ }^{1}$ Department of Clinical Neuroscience

${ }^{2}$ Department of Neuroscience

The worst performance rule (WPR) claims that subjects' worst performance on multi-trial tasks, e.g. in reaction time, is more predictive of their general intelligence than better performances. A common interpretation of the WPR has been that it occurs because poor performances reflect momentary lapses in attention and executive control, which occur more frequently in individuals with low IQ. However, the present simulation study indicates that WPR like phenomena may arise whenever certain statistical relations between the intra-individual variance of multiple test scores and other measures of interest are present. Specifically, we propose that the WPR is a special case of a more general correlation of sorted scores rule (CSSR). According to the CSSR, a WPR (i.e. a negative correlation between the test score percentile of sorted scores, e.g. in reaction time, and the test score $\times$ construct (e.g. g) correlation), will be seen if the intraindividual variance on the tests has a negative correlation with the construct. However, if the latter correlation is positive, the CSSR predicts a positive association also between test score percentiles and test score $\times$ construct correlations, i.e. a "best performance rule". A way to discriminate between a "true" WPR, which occurs independently of these statistical relations, and the CSSR is suggested.

Key words: correlation of sorted scores rule; intelligence; intra-individual variance; reaction time; simulation; worst performance rule

\section{Introduction}

The worst performance rule (WPR), captures an intriguing phenomenon observed in experiments where subjects perform multiple trials on a task which is correlated with general intelligence $(g)$. If the WPR holds, one will find that once the trials of each participant have been sorted after performance, from best to worst, the magnitude of the betweenparticipant correlation between performance and intelligence is larger, the worse the trial. The WPR could thus also be expressed as an anticipated negative association between the performance rank of the test trials, on the one hand, and their correlation with $g$, on the other hand. In brief, poor performances appear to tell more about intelligence than good performances.

Empirical support for the WPR has been received mainly from the association between $g$, or related constructs, and performance on reaction time (RT) tasks (e.g. Diascro \& Brody, 1993; Kranzler, 1992;
Larson \& Alderton, 1990; Schubert, 2019; Unsworth, Redick, Lakey, \& Young, 2010; Wallert, Ekman, Westman, \& Madison, 2017; but see Ratcliff, Thapar, \& McKoon, 2010; and Salthouse, 1998, for some non-confirming findings). It is detectable primarily for tests high in $g$ saturation (Coyle, 2003; Fernandez, Fagot, Dirk, \& de Ribaupierre, 2014; Rammsayer \& Troche, 2016, however, see Schubert, 2019). A common interpretation of the WPR is that it occurs since poor performances, e.g. slow RTs, tap more into neuropsychological processes of importance for intelligence, than do fast RTs. More specifically, slow RTs have been suggested to reflect attentional lapses, which are more common in people with low intelligence (Coyle, 2003; Jensen, 1992). A related hypothesis is that slow RTs are the consequence of lapses in working memory function, which in turn are also more common in individuals with lower intelligence (Larson \& Alderton, 1990). Coyle (2001, 2003) has speculated about the possible neural basis of the WPR, hypothesizing that individuals with lower intelligence experience more frequent glitches or "errors" in neural processing, and that this has detrimental effects on both cognitive performance and RT. These explanations at different levels are of course not mutually exclusive, since less efficient neural processing may well manifest itself as lapses in working memory or attention.

Notably, these cognitive and neural explanations of the WPR are in principle independent of statistical properties such as the distribution and skewness of RT itself. Coyle (2003) has also claimed that the WPR is unlikely to be explained by statistical artifacts, e.g. variance compression or skewness. However, due to an observed difference between unstandardized and standardized estimates, Frischkorn, Schubert, Neubauer, and Hagemann (2016) recently concluded that an increase in the inter-individual standard deviation from best to worst performance may play a crucial role for the WPR.

Another putative explanation of the WPR is based on Ratcliff's influential Diffusion Model of reaction time (Ratcliff, 1978; Ratcliff \& McKoon, 2008). According to this model, information toward one of two decisions, e.g. in a 2-choice reaction time task, is accumulated over time and the neural decision process in the brain of the actor moves toward the correct or incorrect response with a certain speed. This model predicts the commonly observed positive skewness of reaction times. The model also fits with the commonly observed negative association between intelligence and intraindividual mean and variance on reaction time tasks. In a simulation study, Ratcliff, Schmiedek, and McKoon (2008) showed that the diffusion model predicts the WPR, assuming that people high in intelligence accumulate information toward a 
decision at a higher rate, or require a lower amount of information before making a decision, compared to those lower in intelligence.

The main aim of the present study was to use simulations to explicitly test whether WPR-like phenomena can arise as a consequence of statistical properties of RT and its relation to intelligence. Specifically, we were interested in exploring the potential contribution of the empirically wellsupported properties that not only the central tendency of RT but also its intra-individual variance correlates negatively with intelligence, i.e. individuals with higher intelligence tend to have less variable RTs (Doebler \& Scheffler, 2016; Jensen, 1992, 2006). Here, we simulated a number of datasets with RT and intelligence data, while systematically manipulating statistical properties of $\mathrm{RT}$ and its relation to intelligence to analyze their potential contribution to the WPR.

\section{Method}

Using R 3.5.0 software (R Core Team, 2018) a function was created (script available at https://osf.io/6brnj/). This function assigns a specified number of virtual subjects a specified number of test scores (we used 21 scores in our analyses) drawn from a specified beta distribution (we used alpha $=2$ and beta $=9$, which corresponds to a skewness of 0.88), and with a specified approximate correlation between test scores (we used .80). These test scores are sorted, from lowest to highest, for each subject. The intra-individual standard deviation (IISD) and intra-individual mean (IIM) is calculated for each subject. A $g$ score with a specified approximate correlation with IISD and IIM is assigned to each subject. The Spearman's correlation between each of the sorted test scores and $g$ is calculated. The Spearman's correlation between test score percentiles and this vector of Spearman's

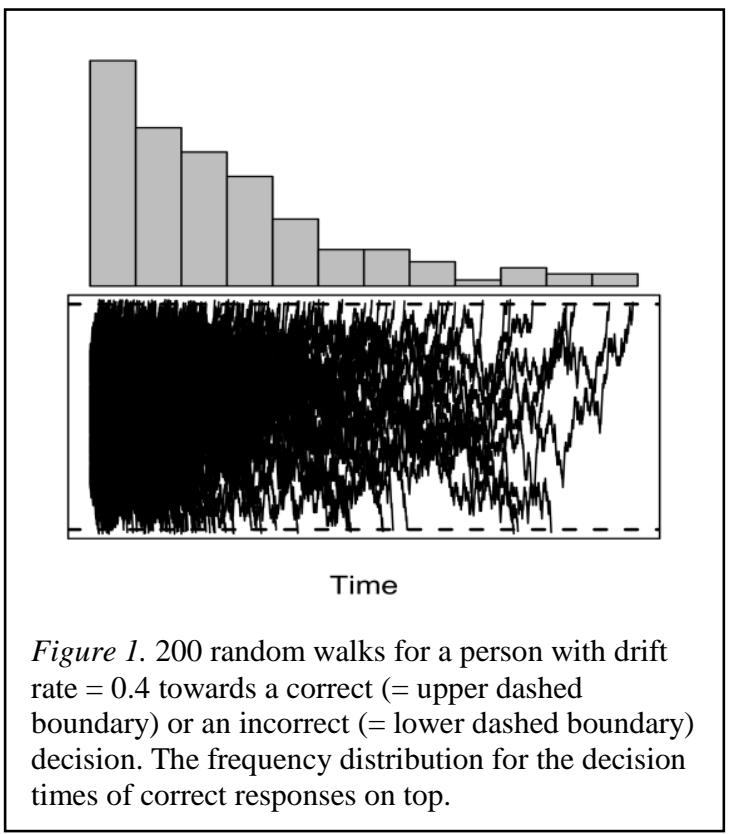

correlations between test scores and $g$ is calculated. This last correlation will hereafter be called the WPR-correlation. A negative WPR-correlation indicates that tests with higher scores, i.e. worse performances on RT tasks, have a more negative correlation with $g$ compared to tests with lower scores.

At the best of our ability, we also tried to replicate Ratcliff et al.s' (2008) simulation of the diffusion process (to be specific, the one on row eight in their Table 1). A thousand virtual subjects were sent out on 200 random walks each, corresponding to reaction time trials, towards one of two decisions and the reaction time was recorded (Figure 1). Average "boundary separation" (the distance between the two dashed boundaries in Figure 1) and "drift rate" (the ability to extract high quality information at high speed, corresponding to intelligence) were varied between subjects. The starting point of the decision process, as well as drift rate, were also varied between trials within subjects. In a random walk, each step (of a constant length between and within subjects) takes the subject either towards the correct or an incorrect decision, and the higher the drift rate the higher the probability (which varies both between and within subjects, but is constant during a specific walk) of a step in the right direction. When the path reaches one of the two decision boundaries, the $\mathrm{x}$-coordinate corresponds to the decision time for that specific trial.

\section{Results}

In Figure 2 we see the association between test score percentiles and the test's Spearman's correlation with $g$ when using sample size 5000, separately for four different combinations of

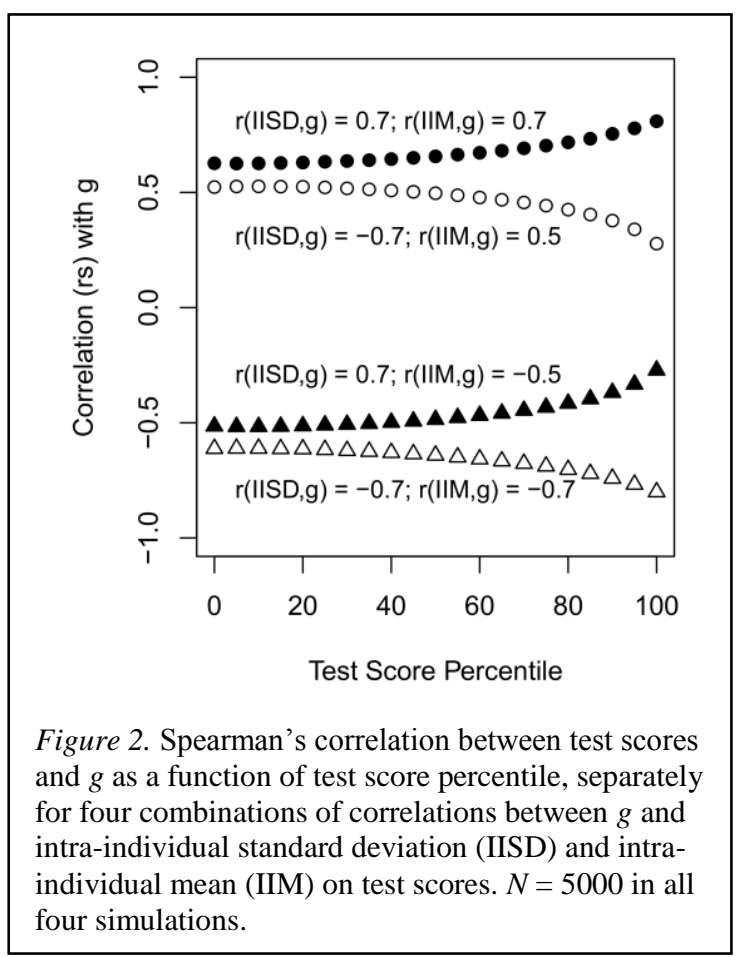



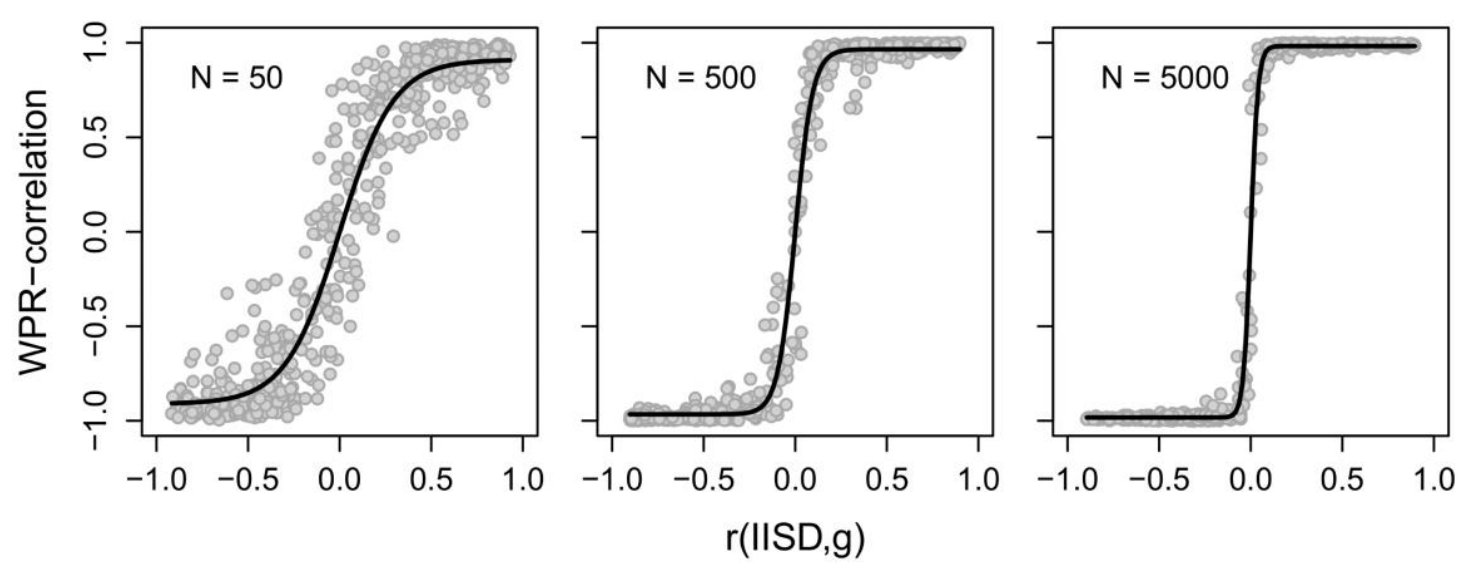

Figure 3. The Spearman's correlation between test score percentile and the vector of Spearman's correlations between test scores and $g(=$ WPR-correlation $)$ as a function of the correlation between the intra-individual standard deviation (IISD) on the tests and $g$, separately for three different sample sizes. 500 simulations for each sample size.

correlations between $g$ and IISD and IIM. The plot indicates that the average correlation between test scores and $g$ is to a high degree defined by the correlation between IIM and $g$ - they are both either positive or negative. We also see that with a positive correlation between IISD and $g$, the correlations between test scores and $g$ tend to increase with test score percentile, and vice versa if the correlation between IISD and $g$ is negative.

Given the findings above, it is not surprising that we found an association between the correlation between IISD and $g$ and the WPR-correlation. With a large sample size the association is more or less dichotomous, with the WPR-correlation approaching 1 if the correlation between IISD and $g$ is positive, and with the WPR-correlation approaching - 1 if it is negative (Figure 3).

As outlined above, the diffusion process was simulated through 200 random walks among 1000 subjects. The reaction times of correct responses were sorted from fastest to slowest within subjects and their association with individual drift rate, corresponding to intelligence, was in line with the WPR (Figure 4). However, when adjusting for differences in IISD in reaction time, the association changed to what could be described as a "best performance rule", with the association between drift rate and reaction time decreasing from a moderately strong positive one to approximately zero with test score percentile. The standardized effect of drift rate (dr) on reaction time (rt) while adjusting for differences in IISD is given by the formula (Cohen, Cohen, West, \& Aiken, 2003):

$$
\beta_{d r, r t . i i s d}=\frac{r_{d r, r t}-r_{i i s d, r t} \cdot r_{i i s d, d r}}{1-r_{i i s d, d r}^{2}}
$$

At low test score percentiles, the association between drift rate and reaction time is close to zero and as the association between IISD and reaction time is positive, while the association between IISD and drift rate is a negative constant $(r=-0.410)$, the adjusted effect of drift rate on reaction time becomes positive. However, with increased test score percentile the association between drift rate and reaction time decreases while the association between IISD and reaction time increases. At the highest test score percentiles, i.e. the worst performances, the association between drift rate and reaction time equals approximately the product of the association between IISD and reaction time and the association between drift rate and IISD, which results in an adjusted effect close to zero. In summary, this indicates that the diffusion model gives results in accordance with the WPR because it produces data where IISD has a negative correlation with drift rate/intelligence.

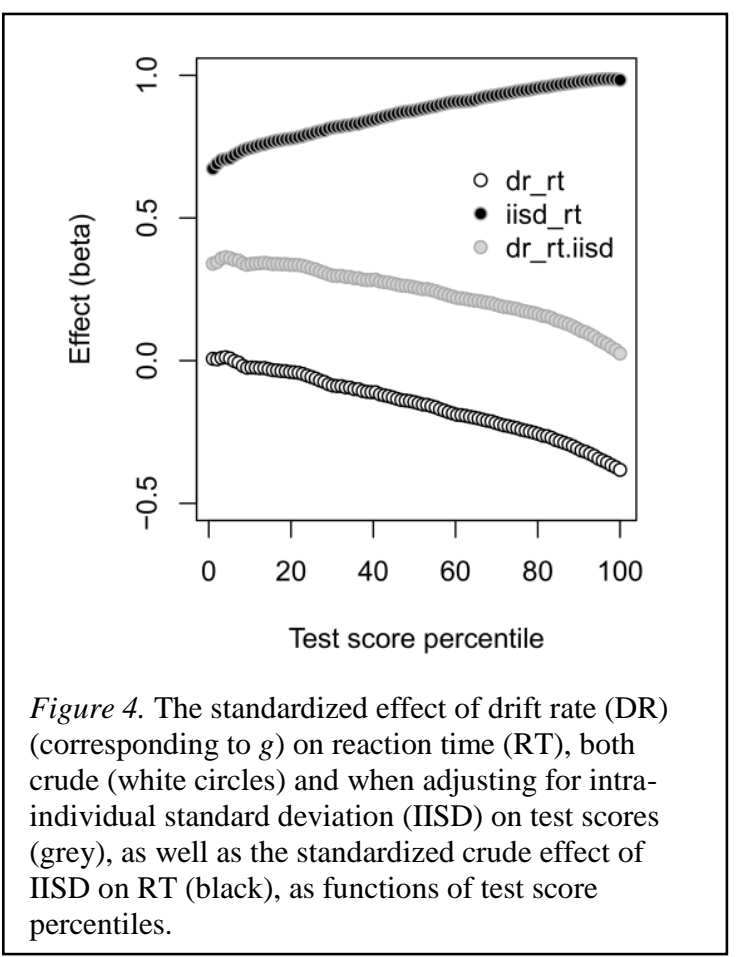




\section{Discussion}

Is the WPR as a special case of the Correlation of Sorted Scores Rule (CSSR)?

The results of the simulations conducted here clearly indicate that WPR like phenomena may appear purely as a consequence of statistical properties of the involved measurements. More specifically, the findings in this simulation study suggest what we propose to call the Correlation of Sorted Scores Rule (CSSR): If subjects take a number of tests (e.g. in reaction time or environmental concern) and the intra-individual variance on these tests has a correlation with a construct $\mathrm{C}$ (e.g. general intelligence or recycling behavior) then the correlation between sorted test scores and $\mathrm{C}$ will increase (i.e. become either more positive or less negative) with increasing test score percentile (from lowest to highest score) if the correlation between the intra-individual variance and $\mathrm{C}$ is positive and the correlation between sorted test scores and $\mathrm{C}$ will decrease (become less positive or more negative) with increasing test score percentile if the correlation between the intra-individual variance and $\mathrm{C}$ is negative. The CSSR is accentuated by a large sample size.

The empirically observed worst performance rule (WPR) may thus be a special case of the CSSR, expected to hold whenever the intra-individual mean and variance on the tests have a negative correlation with the construct (see lowest row of triangles in Figure 2). In fact, both these characteristics are commonly observed in empirical studies when the tests measure performance on reaction time tasks and when the construct is general intelligence (Jensen, 1992).

The CSSR and alternative explanations of the WPR

One can also note that the CSSR is perfectly consistent with previous modelling studies based on Ratcliff's Diffusion Model. Ratcliff et al. (2008) showed that the Diffusion Model predicts the WPR, provided that one of two psychologically plausible conditions hold, i.e. that there is either (a) a positive correlation between intelligence and the model's rate of accumulation of information parameter or (b) a negative correlation between intelligence and a parameter deciding the amount information needed before taking a decision. Here, we replicated a simulation of the Diffusion Model. Importantly, we additionally demonstrate that the WPR exhibited by this model vanishes when controlling statistically for intra-individual variance in RT. The Diffusion Model thus appears to produce a WPR because it produces data that has the statistical properties - i.e. dependencies between test score variance and intelligence - required for the CSSR. This observation is of course in no way an invalidation of the diffusion model, which has been remarkably successful in capturing many phenomena relating to RT tasks, but clarifies which properties of the Diffusion Model that are critical for explaining the WPR.

Secondly, the CSSR may also in principle be compatible with some of the psychological and neural explanations of the WPR that have been proposed. The implication of the CSSR is not necessarily that the WPR is "only an artifact", as the negative correlation between intra-individual mean and variance on reaction time performance and $g$, and maybe also to some extent the commonly observed positive skewness of the performances, could be caused by some of the neuropsychological factors that have been suggested as explanations of the WPR, for example that occasional long reaction times are caused by lapses in attention and working memory and that such lapses are experienced to a higher degree by subjects low in $g$ (Jensen, 1992; Larson \& Alderton, 1990; Unsworth et al., 2010; see Coyle, 2003, for a review).

In future studies it would be important to test the CSSR empirically. A critical distinction between the CSSR and the WPR is that they give different predictions in a situation where test score variance is positively related to intelligence. A critical test could therefore be to construct a paradigm where subjects mainly perform badly on the used tests, but occasionally the brightest of them "sees the light" and get a good score, resulting in a positive correlation between intra-individual variance and $g$. In this case a bona fide WPR would still predict that worst performances have the strongest correlation with $g$, while the CSSR would predict the opposite. May the best rule win.

\section{References}

Cohen, J., Cohen, P., West, S. G., \& Aiken, L. S. (2003). Applied Multiple Regression/Correlation Analysis for the Behavioral Sciences. Mahwah, NJ: Lawrence Erlbaum Associates.

Coyle, T. R. (2001). IQ is related to the worst performance rule in a memory task involving children. Intelligence, 29, 117-129.

Coyle, T. R. (2003). A review of the worst performance rule: Evidence, theory, and alternative hypotheses. Intelligence, 31, 567-587.

Diascro, M. N., \& Brody, N. (1993). Serial versus parallel processing in visual search tasks and IQ. Personality and Individual Differences, 14, 243-245.

Doebler, P., \& Scheffler, B. (2016). The relationship of choice reaction time variability and intelligence: A meta-analysis. Learning and Individual Differences, 52, 157-166.

Fernandez, S., Fagot, D., Dirk, J., \& de Ribaupierre, A. (2014). Generalization of the worst performance rule across the lifespan. Intelligence, 42, 31-43.

Frischkorn, G. T., Schubert, A.-L., Neubauer, A. B., \& Hagemann, D. (2016). The worst performance rule as moderation: New methods for worst performance analysis. Journal of Intelligence, 4, 9. 
Jensen, A. R. (1992). The importance of intraindividual variation in reaction time. Personality and Individual Differences, 13, 869-881.

Jensen, A. R. (2006). Clocking the mind: mental chronometry and individual differences. Oxford, UK: Elsevier.

Kranzler, J. H. (1992). A test of Larson and Alderton's (1990) worst performance rule of reaction time variability. Personality and Individual Differences, $13,255-261$

Larson, G. E., \& Alderton, D. L. (1990). Reaction time variability and intelligence: A "worst performance" analysis of individual differences. Intelligence, 14, 309-325.

R Core Team (2018). $R$ : A language and environment for statistical computing. R Foundation for Statistical Computing, Vienna, Austria. URL https://www.Rproject.org/.

Rammsayer, T. H., \& Troche, S. J. (2016). Validity of the worst performance rule as a function of task complexity and psychometric g: On the crucial role of g saturation. Journal of Intelligence, 4, 5.

Ratcliff, R. (1978). A theory of memory retrieval. Psychological Review, 85, 59-108.
Ratcliff, R. \& McKoon, G. (2008). The diffusion decision model: Theory and data for two-choice decision tasks. Neural Computation, 20, 873-922.

Ratcliff, R., Schmiedek, F., \& McKoon, G. (2008). A diffusion model explanation of the worst performance rule for reaction time and IQ. Intelligence, 36, 10-17.

Ratcliff, R., Thapar, A., \& McKoon, G. (2010). Individual differences, aging, and IQ in two-choice tasks. Cognitive Psychology, 60, 127-157.

Schubert, A.-L. (2019). A meta-analysis of the worst performance rule. Intelligence, 73, 88-100.

Salthouse, T. A. (1998). Relation of successive percentiles of reaction time distributions to cognitive variables and adult age. Intelligence, 26, 153-166.

Unsworth, N., Redick, T. S., Lakey, C. E., \& Young, D. L. (2010). Lapses in sustained attention and their relation to executive control and fluid abilities: An individual differences investigation. Intelligence, 38 , 111-122.

Wallert, J., Ekman, U., Westman, E., \& Madison, G. (2017). The worst performance rule with elderly in abnormal cognitive decline. Intelligence, 64, 9-17. 